\title{
Antimicrobial susceptibility of Neisseria gonorrhoeae isolates from Hefei (2014-2015): genetic characteristics of antimicrobial resistance
}

Fa-Xing Jiang ${ }^{1+}$, Qian Lan ${ }^{1+}$, Wen-Jing Le ${ }^{2}$ and Xiao-Hong Su${ }^{2 *}$

\begin{abstract}
Background: Antimicrobial resistance (AMR) and genetic determinants of resistance of N. gonorrhoeae isolates from Hefei, China, were characterized adding a breadth of information to the molecular epidemiology of gonococcal resistance in China.

Methods: 126 N. gonorrhoeae isolates from a hospital clinic in Hefei, were collected between January, 2014, and November, 2015. The minimum inhibitory concentration (MIC) of N. gonorrhoeae isolates for seven antimicrobials were determined by the agar dilution method. Isolates were tested for mutations in penA and $m$ trR genes and $23 \mathrm{~S}$ rRNA, and also genotyped using N. gonorrhoeae multi-antigen sequence typing (NG-MAST).

Results: All N. gonorrhoeae isolates were resistant to ciprofloxacin; $81.7 \%(103 / 126)$ to tetracycline and $73.8 \%$ (93/126) to penicillin. 39.7\% (50/126) of isolates were penicillinase producing N. gonorrhoeae (PPNG), 31.7\% (40/126) were tetracycline resistant N. gonorrhoeae (TRNG) and 28.6\% (36/126) were resistant to azithromycin. While not fully resistant to extended spectrum cephalosporins (ESCS), a total of 14 isolates (11.1\%) displayed decreased susceptibility to ceftriaxone (MIC $\geq 0.125 \mathrm{mg} / \mathrm{L}, n=10)$, cefixime (MIC $\geq 0.25 \mathrm{mg} / \mathrm{L}, n=1)$ or to both ESCs $(n=3)$. penA mosaic alleles XXXV were found in all isolates that harbored decreased susceptibility to cefixime, except for one. Four mutations were found in mtrR genes and mutations A2143G and C2599T were identified in 23S rRNA. No isolates were resistant to spectinomycin. Gonococcal isolates were distributed into diverse NG-MAST sequence types (STs); 86 separate STs were identified.

Conclusions: N. gonorrhoeae isolates from Hefei during 2014-2015, displayed high levels of resistance to antimicrobials that had been recommended previously for treatment of gonorrhea, e.g., penicillin, tetracycline and ciprofloxacin. The prevalence of resistance to azithromycin was also high (28.6\%). No isolates were found to be fully resistant to spectinomycin, ceftriaxone or cefixime; however, $11.1 \%$ isolates, overall, had decreased susceptibility to ESCs.
\end{abstract}

Keywords: Neisseria gonorrhoeae, Antimicrobial resistance, Azithromycin, Cephalosporins, Resistance determinants

\footnotetext{
* Correspondence: suxh@ncstdlc.org

${ }^{\dagger}$ Equal contributors

${ }^{2}$ STD clinic, Institute of Dermatology, Chinese Academy of Medical Sciences

\& Peking Union Medical College, Nanjing 210042, China

Full list of author information is available at the end of the article
}

(c) The Author(s). 2017 Open Access This article is distributed under the terms of the Creative Commons Attribution 4.0 International License (http://creativecommons.org/licenses/by/4.0/), which permits unrestricted use, distribution, and reproduction in any medium, provided you give appropriate credit to the original author(s) and the source, provide a link to the Creative Commons license, and indicate if changes were made. The Creative Commons Public Domain Dedication waiver (http://creativecommons.org/publicdomain/zero/1.0/) applies to the data made available in this article, unless otherwise stated. 


\section{Background}

Neisseria gonorrhoeae is the etiologic agent of gonorrhea, one of the most common bacterial sexually transmitted infections (STIs) worldwide. WHO estimated that 78.3 million of new cases of gonorrhea occurred among adults globally in 2012 [1].100, 245 cases of gonorrhea were reported nationally by the China Centers for Disease Control and Prevention in 2015, making it the fifth most commonly reported communicable disease in China [2]. At this time there are no effective vaccines for gonococcal infections and antimicrobial treatment continues to be the mainstay of control. However, N. gonorrhoeae has developed resistance to antimicrobials that had been used previously for treatment of this infection including sulfonamides, penicillins, tetracyclines and quinolones. Presently, extended spectrum cephalosporins (ESCs) that include ceftriaxone and cefixime are recommended as the first-line treatment of gonorrhea in most parts of the world. In many countries azithromycin has been added not just to treat chlamydia infection that often co-infects, but also to supplement treatment for $N$. gonorrhoeae itself [3]. Diminished susceptibility of $N$. gonorrhoeae and emergence of full resistance and treatment failures with ESCs (mainly pharyngeal gonorrhea) have been documented in several countries [4]. Furthermore, N. gonorrheae strains with high-level azithromycin resistance have been reported in France [5], the United States [6], Australia [7] and China [8]. Resistance to azithromycin threatens efficacy of dual antimicrobial therapy (ESCs plus azithrothmycin) that may result in decreased treatment options and enhance the possibility of untreatable infection.

A key component of a successful response plan to gonococcal antimicrobial resistance (AMR) is to conduct timely surveillance of resistance and treatment failures across geographic regions (e.g. GISP [U.S] and GASP (WHO/Europe) $[9,10]$ ) and to characterize the genetic elements of resistant strains. Antimicrobial susceptibility patterns vary by geographic region. AMR surveillance programs have been conducted in several cities in China $[11,12]$. The aims of the present study were to describe the prevalence of gonococcal AMR and the molecular epidemiological characteristics of $N$. gonorrhoeae strains from 2014 to 2015 in Hefei, a city in eastern China.

\section{Methods}

\section{Clinical isolates}

Clinical isolates of $N$. gonorrhoeae $(n=126)$ investigated in this study were collected consecutively from men with urethritis (urethral discharge and/or dysuria) and women with cervical infection in the STD clinic at Anhui Provincial Hospital, China, between January, 2014 and November 2015. Cotton swabs, used to obtain cervical and urethral specimens, were immediately streaked onto
Thayer-Martin (T-M) selective medium to isolate $N$. gonorrhoeae. Inoculated plates were incubated at $36{ }^{\circ} \mathrm{C}$ in 5\% carbon dioxide for 24-48 h. and $N$. gonorrhoeae was identified by colonial morphology, Gram's stain, and oxidase testing. Gonococcal strains were subcultured onto chocolate agar plates, preserved in tryptone-based soy broth and then stored at $-70{ }^{\circ} \mathrm{C}$ until used.

\section{Antimicrobial susceptibility testing}

Minimum inhibitory concentrations (MICs; mg/L) of $N$. gonorrhoeae isolates to penicillin, tetracycline, ciprofloxacin, spectinomycin, azithromycin, ceftriaxone and cefixime were determined on Difco GC medium base agar supplemented with $1 \% \mathrm{BBL}^{\mathrm{Tm}}$, IsoVitaleX ${ }^{\mathrm{mm}}$ enrichment (Becton, Dickinson and Company) using the agar dilution method recommended by the Clinical and Laboratory Standards Institute (CLSI) [13]. Gonococcal isolates were subcultured from frozen stocks onto chocolate agar and the resulting colonies were re-subcultured at $36^{\circ} \mathrm{C}$ in $5 \% \mathrm{CO}_{2}$ for $18-20 \mathrm{~h}$ before antimicrobial susceptibility testing was performed. Concentrations of antibiotics used were: penicillin, $0.06-8 \mathrm{mg} / \mathrm{L}$; tetracycline, $0.125-$ $16 \mathrm{mg} / \mathrm{L}$; ciprofloxacin, 0.06-8 $\mathrm{mg} / \mathrm{L}$; spectinomycin, 4-128 mg/L; azithromycin,0.015-2048 mg/L; ceftriaxone, $0.002-0.5 \mathrm{mg} / \mathrm{L}$ and cefixime,0.002-0.5 mg/L. All antibiotics were purchased from Sigma Aldrich (USA), except for azithromycin, which was purchased from Shanghai yuanye Bio-Technology Co., Ltd. 95\% ethanol was used to dissolve azithromycin powder to obtain a stock solution of $4096 \mathrm{mg} / \mathrm{L}$; azithromycin stock was further diluted with distilled water to prepare twofold working dilutions. ATCC49226 and WHO reference strains $\mathrm{G}, \mathrm{K}, \mathrm{M}, \mathrm{O}, \mathrm{P}$ were used as quality controls for MIC determinations. Results were interpreted according to the CLSI standard, except for azithromycin that used breakpoints recommended by the European Committee on Antimicrobial Susceptibility Testing (EUCAST); www.eucast.org [14].Criteria for decreased susceptibility to ceftriaxone (MIC $\geq 0.125 \mathrm{mg} / \mathrm{L}$ ) and cefixime $(\mathrm{MIC} \geq 0.25 \mathrm{mg} / \mathrm{L})$ were defined by WHO [9].

\section{Identification and typing of $\beta$-lactamase and tet $M$ encoding plasmids}

$\beta$-lactamase production was determined by the paper acidometric method [15]. Isolates were classified as high level resistant to tetracycline (TRNG) if MICs were $\geq 16 \mathrm{mg} / \mathrm{L}$ and resistant (plasmid-mediated) to penicillin (PPNG) if they were $\beta$-lactamase positive [16]. The type of $\beta$-lactamase and tet $M$ encoding plasmids were determined by PCR [17]. 
Genetic determinants associated with decreased susceptibility to ESCs and resistance of $N$. gonorrhoeaeto azithromycin

Amplification of pen $A$ and $m$ trR genes and 23S rRNA were performed using published primers and conditions [18-20]. 14 gonococcal isolates with decreased susceptibility to ceftriaxone or cefixime were tested for penA mutations, including 3 isolates that had decreased susceptibility to both cefixime and ceftriaxone; all isolates were tested for mutations in $m t r R$ and 41 strains (including all 36 azithromycin-resistant and 5 randomly selected azithromycin sensitive strains) were tested for 23S rRNA mutations. PCR products were sequenced twice in both directions using an Applied Biosystems 3730XL DNA automatic sequencer. The nucleotide and deduced amino acid sequences were analyzed using the EditSeq program (LaserGene software [version 7.1; DNAStar Corp.]) and aligned against their respective prototypes using the Megalign program (LaserGene software). Standard sequences used for alignment in the study were: PBP2; wild strain LM306 (GenBank accession no. M32091 [18]); 4 alleles of 23S rRNA (GenBank accession no. AF450074 to AF450081 [20]) and mtrR (GenBank accession no. Z25796 [19]).

\section{Molecular epidemiologic typing}

$N$. gonorrhoeae multiantigen sequence typing (NGMAST) was performed on all isolates $(n=126)$ as described [21]. The allele numbers of por and $t b p B$, and the sequence types (STs) were assigned using the NG-MAST website (www.ng-mast.net).

\section{Statistical analysis}

Statistical analysis was performed using statistical software IBM SPSS Statistics version 19.0 for comparisons of proportions. The level of significance was set at $P<0.05$.

\section{Results}

Antimicrobial susceptibility testing

Antimicrobial susceptibility testing of 126 gonococcal isolates obtained from Hefei, China, between January, 2014 and November, 2015, is indicated in Table 1. Resistance to penicillin and tetracycline was $73.8 \%$ (93/ $126)$ and $81.7 \%$ (103/126), respectively; all isolates were resistant to ciprofloxacin. $28.6 \%(36 / 126)$ of isolates were resistant to azithromycin; among them,36.1\% (13/36) displayed high-level azithromycin resistance (MIC $\geq 256 \mathrm{mg} / \mathrm{L})$. No isolates were found to be fully resistant to spectinomycin, ceftriaxone or cefixime. However,14 isolates (11.1\%) displayed decreased susceptibility to ceftriaxone (MIC $\geq 0.125 \mathrm{mg} / \mathrm{L}, n=10)$, cefixime (MIC $\geq 0.25 \mathrm{mg} / \mathrm{L}, n=1)$ or to both ESCs $(n=3)$.Two azithromycin-resistant isolates also exhibited reduced susceptibility to ceftriaxone. Overall, plasmid mediated resistance to either penicillin or tetracycline was exhibited by $60.3 \%(76 / 126)$ of isolates: $39.7 \% \quad(50 / 126)$ were PPNG; $31.7 \%$ (40/126) were TRNG and $11.1 \%(14 / 126)$ were PPNG/TRNG. $80 \%$ (40/50) PPNG isolates carried the Asia type $\beta$ lactamase encoding plasmid and 20\% (10/50) harbored the African type plasmid. No Toronto plasmid was detected. 97.5\% (39/40) of TRNG isolates carried the Dutch type tet $M$ containing plasmid and the remainder carried the American-type.

\section{Characterization of resistance in pen $A$ and $m t r R$ genes and 235 rRNA \\ Mutations in the penA gene}

We sequenced the penA gene from 14 isolates that displayed decreased susceptibility to ceftriaxone $(n=10)$, cefixime $(n=1)$ or to both ESCs $(n=3)$ (Table 2$)$. Six PBP2 amino acid sequence patterns were identified, including a mosaic allele (XXXV) [18]. PBP2 allele XIII [19] was the predominant type $(n=5)$, followed by mosaic allele XXXV $(n=3)$ and alleles XVIII $(n=3), \mathrm{V}$ $(n=1)$, XVII $(n=1)$ and XXI $(n=1)[19,22,23]$. The three isolates that harbored mosaic allele XXXV had decreased susceptibility to cefixime; one (of the 3) displayed decreased susceptibility to both ceftriaxone and cefixime. Among 11 non-mosaic isolates that included 10 with decreased susceptibility to ceftriaxone and 1 to both ESCs, seven harbored alleles XIII, XVII and XXI and displayed an A501V mutation in PBP2. Three isolates with allele XVIII possessed an A501T mutation and one had a G542S mutation.

\section{Mutations in the mtrR gene}

All 126 isolates had mutations in the $m t r R$ gene. Adenine $(\mathrm{A})$ was deleted in the promoter region of $m t r R$ in $107(87.3 \%)$ strains; there was no difference $(p=0.181)$ in isolates with MICs $\geq 1 \mathrm{mg} / \mathrm{L}$ to azithromycin $(33 / 36$ [91.7\%]) compared to isolates with an $\mathrm{MIC} \leq 0.5 \mathrm{mg} / \mathrm{L}$ (74/90 [82.2\%]). However, the G45D mutation in the $m t r R$ gene was identified more often in azithromycinresistant isolates $(25 / 36[69.4 \%])$ than in susceptible isolates $(9 / 90[10 \%])(p<0.01)$. The A40D mutation was present in three isolates with (lower) azithromycin MICs of $0.125 \mathrm{mg} / \mathrm{L}$; these isolates also exhibited reduced cefixime and/or ceftriaxone susceptibility. The A39T mutation was found in 16 more sensitive isolates, having MICs to azithromycin $>0.125 \mathrm{mg} / \mathrm{L}$ but $<0.5 \mathrm{mg} / \mathrm{L}$.

\section{Mutations in domains of 235 rRNA}

All 36 azithromycin-resistant isolates and five -susceptible isolates were tested for mutations in 23S rRNA. The A2143G ( $N$. gonorrhoeae numbering) mutation was identified in four alleles in the13 isolates with high-level 
Table 1 Antimicrobial susceptibility of Neisseria gonorrhoeae isolates ( $n=126)$ from Hefei, China (2014-2015)

\begin{tabular}{|c|c|c|c|c|c|c|}
\hline \multirow{2}{*}{$\begin{array}{l}\text { Antimicrobial breakpoints } \\
\text { (susceptible/resistant [mg/L]) }\end{array}$} & \multicolumn{3}{|l|}{ Number } & \multicolumn{3}{|l|}{ MIC (mg/L) } \\
\hline & Susceptible & Intermediate & Resistant & Range & $\mathrm{MIC}_{50}$ & $\mathrm{MIC}_{90}$ \\
\hline Penicillin $G(S \leq 0.06 / R \geq 2)$ & 0 & 33 & 93 & 0.125 to $>8$ & 2 & $>8$ \\
\hline Tetracycline $(S \leq 0.25 / R \geq 2)$ & 2 & 21 & 103 & 0.25 to $>16$ & 4 & $>16$ \\
\hline Ciprofloxacin ( $S \leq 0.06 / R \geq 1)$ & 0 & 0 & 126 & 0.25 to $>8$ & $>8$ & $>8$ \\
\hline Azithromycin $(S \leq 0.25 / R \geq 1)$ & 48 & 42 & 36 & $\leq 0.015$ to $>2048$ & 0.5 & $>2048$ \\
\hline Spectinomycin $(S \leq 32 / R \geq 128)$ & 126 & 0 & 0 & 8 to 32 & 16 & 32 \\
\hline Cefixime $(S \leq 0.25)$ & 126 & 0 & 0 & 0.004 to 0.25 & 0.03 & 0.06 \\
\hline Ceftriaxone( $(\mathrm{S} \leq 0.25)$ & 126 & 0 & 0 & 0.004 to 0.25 & 0.06 & 0.125 \\
\hline
\end{tabular}

MIC minimum inhibitory concentration

azithromycin resistance (MICs $\geq 256 \mathrm{mg} / \mathrm{L}$ ). AC259 $9 \mathrm{~T}$ mutation in four alleles was found in four isolates (MICs, 8 to $32 \mathrm{mg} / \mathrm{L}$ ).No mutations were found in the five azithromycin-susceptible isolates.

\section{Sequence-based molecular epidemiologic typing}

The $126 \mathrm{~N}$. gonorrhoeae isolates were assigned to 86 different NG-MASTs (abbreviated henceforth as STs); 53 (61.6\%) STs were novel. 19 STs were shared by $\geq 2$ isolates and $67 \mathrm{STs}$ were represented only in single isolate. The most common ST was7469, represented by 6 isolates (4.8\%), followed by ST1866 (5 isolates; 4.0\%). All ST7469 isolates were comprised exclusively of the Dutch-type TRNG; all ST1866 isolates were resistant to azithromycin (MIC $\geq 1 \mathrm{mg} / \mathrm{L}$ ). Three isolates, which possessed the penA mosaic allele XXXV and had decreased susceptibility to cefixime (MIC $=0.25 \mathrm{mg} / \mathrm{L}$ ), belonged to different STs (ST12200, ST12647, ST13044) but shared identical $t b p B 10$.

\section{Discussion}

A high prevalence of resistance to antimicrobials previously used for the treatment of gonorrhea was documented in Hefei (2014-15) in this study: penicillin (68.9\%); tetracycline (80.6\%) and ciprofloxacin (100\%). Similar levels of resistance to ciprofloxacin, tetracycline and penicillin have been reported in N. gonorrhoeae isolates from other cities in China: Nanjing (penicillin $67.7 \%$, tetracycline $97.9 \%$ and ciprofloxacin $98.8 \%$, in 2011-2012) [17]; Shanghai (penicillin 90\%, tetracycline $82.56 \%$ and ciprofloxacin 100\%, in 1988-2013) [11] and Guangzhou (penicillin $90.1 \%$ and ciprofloxacin $98 \%$, in 2008-2013) [12]. A high percentage of PPNG and TRNG isolates was also identified in Hefei. Because penicillin and tetracycline are no longer used for the treatment of gonorrhea in China, continued high resistance may have been the result of having used these antimicrobials to treat other infections, particularly in patients who self-medicate, which is common in China [24].
Resistance to azithromycin was $28.6 \%$, higher than had been reported from certain other countries, such as Japan [25] and Spain [26] during this period. We found that $10.3 \%(13 / 126)$ of isolates were highly resistant to azithromycin (MIC > $2048 \mathrm{mg} / \mathrm{L}$ ). N. gonorrhoeae isolates with high-level azithromycin resistance have also been reported from other Chinese cities; Hanghzou [27] and Guangzhou [28]. Similar to other reports [29, 30], our isolates with high-level azithromycin resistance all contained the mutation A2143G in 4 alleles in the peptidyltransferase loop of domain $\mathrm{V}$ of $23 \mathrm{~S}$ rRNA. The C2599T mutation was found in $23 \mathrm{~S}$ rRNA alleles in four moderately azithromycin-resistant $N$. gonorrhoeae isolates (representing MICs of 8,16 , and $32 \mathrm{mg} / \mathrm{L}$ ).

There are no reports of ceftriaxone treatment failures in China. The percentage of isolates with decreased susceptibility to ceftriaxone (MIC $\geq 0.125 \mathrm{mg} / \mathrm{L}$ ) was $10.3 \%$

Table 2 Characteristics of $N$ gonorrhoeae isolates $(n=14)$ with increased MICs to ceftriaxone (MIC, $0.125 \mathrm{mg} / \mathrm{L}$ ) or cefixime (MIC, $0.25 \mathrm{mg} / \mathrm{L})$

\begin{tabular}{|c|c|c|c|c|}
\hline \multirow{2}{*}{$\begin{array}{l}\text { Strain } \\
\text { number }\end{array}$} & \multicolumn{2}{|l|}{$\mathrm{MIC}(\mathrm{mg} / \mathrm{L})$} & \multirow{2}{*}{$\begin{array}{l}\text { PBP2 } \\
\text { allele }\end{array}$} & \multirow{2}{*}{$\begin{array}{l}\text { NG- } \\
\text { MAST }\end{array}$} \\
\hline & Ceftriaxone & $\overline{\text { Cefixime }}$ & & \\
\hline HF17 & 0.125 & 0.06 & $X V I I I$ & ST12197 \\
\hline HF20 & 0.125 & 0.25 & XIII & ST1577 \\
\hline HF37 & 0.125 & 0.25 & $X X X V^{a}$ & ST12200 \\
\hline HF 42 & 0.125 & 0.125 & $X I I I$ & ST12192 \\
\hline HF 109 & 0.06 & 0.25 & $X X X V^{a}$ & ST12647 \\
\hline HF 141 & 0.125 & 0.125 & XIII & ST12662 \\
\hline HF 149 & 0.125 & 0.125 & XIII & ST12192 \\
\hline HF 152 & 0.125 & 0.06 & V & ST12198 \\
\hline HF 154 & 0.125 & 0.06 & $X I I I$ & ST 2318 \\
\hline HF 156 & 0.125 & 0.25 & $X X X V^{a}$ & ST13044 \\
\hline HF 163 & 0.125 & 0.06 & $X V I I I$ & ST3356 \\
\hline HF 169 & 0.125 & 0.06 & $\mathrm{XVII}$ & ST13133 \\
\hline HF 170 & 0.125 & 0.125 & $X X I$ & ST13134 \\
\hline HF 175 & 0.125 & 0.06 & $X V I I I$ & ST12479 \\
\hline
\end{tabular}

${ }^{\mathrm{a}}$ Mosaic penA alleles

${ }^{\mathrm{b}}$ Novel STs 
(13/126) in Hefei in 2014-2015, which is higher than the corresponding 4.5\% reported from another eastern Chinese city, Nanjing, between 2011 and 2012 [17]. The proportions of ceftriaxone non-susceptible isolates (MIC $\geq 0.125 \mathrm{mg} / \mathrm{L}$ ) in Shanghai, the largest city in eastern China, were 7\%-13\% from 2011 to 2013 [11]. This suggests the possibility that resistance to extended spectrum cephalosporins (ESCs) may be increasing in eastern China. PBP2 is targeted by cephalosporins and mutations in PBP2 may lead to a decline in susceptibility to ESCs [31]. Four (3.2\%) of our isolates had reduced susceptibility to cefixime $(\mathrm{MIC}=0.25 \mathrm{mg} / \mathrm{L})$; three (ST12200, ST12647, ST13044) had the mosaic allele XXXV. The majority (7/11 [63.6\%]) of non-mosaic isolates with decreased susceptibility to ceftriaxone harbored mutations in PBP2 at position 501 (A501V or A501T). Other studies have also found that A501 mutations in non-mosaic penA alleles play an important role in decreased sensitivity to ESCs [32]. A G542S mutation in PBP2 has been tentatively linked epidemiologically to higher ceftriaxone MICs [33] but an absolute correlation with a penA fixed-point mutation has not been shown. ST1407 N. gonorrhoeae strains, which display decreased susceptibility to ESCs or full resistance [34], and have spread worldwide, were not identified in Hefei. Five different STs (ST10367, ST12649, ST12650, ST12657, ST13132) that shared the same $\operatorname{tbpB110}$ with ST1407 were identified but their por alleles were different (by 13 to 37 nucleotides) than por 908 of ST1407; all isolates with these STs were susceptible to both ceftriaxone and cefixime. ST1407, however, has been isolated in Shanghai [35].

Significant diversity of isolates was identified among 126 isolates that were divided into 86 NG-MAST STs. Notably, ST1866 has been reported only from China and identified to be associated with high level azithromycin resistance [28]. The 5 ST1866 isolates in this study were azithromycin-resistant strains; three had high level resistance to azithromycin.

\section{Conclusions}

This is the first study that describes antimicrobial resistance profiles and molecular determinants of $N$. gonorrhoeae strains isolated from Hefei, China. $N$. gonorrhoeae isolates from Hefei, during 2014-2015, possessed high level resistance to antimicrobials previously recommended for the treatment of gonorrhea, e.g., penicillin, tetracycline and ciprofloxacin. Azithromycin-resistance was documented in $28.6 \%$ of isolates, higher than that reported from other areas of the world. No isolates were found to be resistant to ceftriaxone, cefixime or spectinomycin. However, $11.1 \%$ isolates had decreased susceptibility to ESCs, which was often associated with mutations in the penA gene. Gonococcal isolates were distributed into diverse NG-MAST sequence types.

\section{Abbreviations}

AMR: Antimicrobial resistance; CLSI: Clinical and Laboratory Standards Institute; ESCs: Extended-spectrum cephalosporins; GASP: Gonococcal antimicrobial surveillance programme; GISP: Gonococcal Isolate Surveillance Project; MIC: Minimum inhibitory concentration; NG-MAST: Neisseria gonorrhoeae multi-antigen sequence typing; PBP: Penicillin-binding protein; PPNG: Penicillinase-producing N. gonorrhoeae; ST: Sequence type;

STD: Sexually transmitted disease; STI: Sexually transmitted infection;

TRNG: Tetracycline-resistant N. gonorrhoeae; WHO: World Health Organization

\section{Acknowledgements}

We thank Dr. Na Wang for assistance in collection of gonococcal isolates, Dr. Magnus Unemo for providing WHO reference strains and Dr. Peter A. Rice for assistance in writing the manuscript.

\section{Funding}

This study was supported by Nature Science foundation of Anhui Province (No. 090413143).

\section{Availability of data and materials}

The authors declare that the novel sequence types(STs) of Neisseria gonorrhoeae multiantigen sequence typing (NG-MAST) found in this study have been deposited in the NG-MAST website (www.ng-mast.net) and all other data supporting the findings of this study are available within the article.

\section{Authors' contributions}

FXJ co-directed the project and supervised the collection of bacterial isolates. QL assisted with antimicrobial susceptibility testing, performed sequencing, assigned NG-MAST designations and prepared the first draft of the manuscript. XHS also co-directed the project and was involved in preparation of the manuscript. WJL carried out antimicrobial susceptibility testing and identified tet $M$ and $\beta$-lactamase. All authors read and approved the final manuscript.

\section{Competing interests}

The authors declare no competing interests.

\section{Consent for publication}

Not applicable.

Ethics approval and consent to participate

No formal ethics approval was required in this study.

\section{Publisher's Note}

Springer Nature remains neutral with regard to jurisdictional claims in published maps and institutional affiliations.

\section{Author details}

${ }^{1}$ Department of Dermatology, Anhui Provincial Hospital, Hefei 230001, China. ${ }^{2}$ STD clinic, Institute of Dermatology, Chinese Academy of Medical Sciences \& Peking Union Medical College, Nanjing 210042, China.

Received: 24 February 2017 Accepted: 17 May 2017

Published online: 25 May 2017

References

1. Newman L, Rowley J, Vander Hoorn S, Wijesooriya NS, Unemo M, Low N, et al. Global estimates of the prevalence and incidence of four curable sexually transmitted infections in 2012 based on systematic review and Global reporting. PLoS One. 2015;10:e0143304.

2. National health and Family Planning Commision of China. General situation of national legal infectious diseases in 2015. Available at: http://www.nhfpc. gov.cn/jkj/s3578/201602/b9217ba14e17452aad9e45a5bcce6b65. Accessed February 18, 2016

3. Bignell C, Unemo M. European STI Guidelines Editorial Board. 2012 European guideline on the diagnosis and treatment of gonorrhoea in adults. Int J STD AIDS. 2013;24:85-92.

4. Unemo M. Current and future antimicrobial treatment of gonorrhoea - the rapidly evolving Neisseria gonorrhoeae continues to challenge. BMC Infect Dis. $2015 ; 15: 364$ 
5. Bercot B, Belkacem A, Goubard A, Mougari F, Sednaoui P, La Ruche G, Cambau E. High-level azithromycin-resistant Neisseria gonorrhoeae clinical isolate in France, March 2014. Euro Surveill. 2014;19( 44): pii= 20951. Available online: http://www.eurosurveillance.org/ViewArticle.aspx?Articleld= 20951.

6. Katz AR, Komeya AY, Soge OO, Kiaha MI, Lee MV, Wasserman GM, et al. Neisseria gonorrhoeae with high-level resistance to azithromycin: case report of the first isolate identified in the United States. Clin Infect Dis. 2012:54:841-3.

7. Stevens K, Zaia A, Tawil S, Bates J, Hicks V, Whiley D, et al. Neisseria gonorrhoeae isolates with high-level resistance to azithromycin in Australia. J Antimicrob Chemother. 2015;70:1267-8.

8. $\mathrm{Ni} \mathrm{C}$, Xue J, Zhang C, Zhou H, van der Veen S. High prevalence of Neisseria gonorrhoeae with high-level resistance to azithromycin in Hangzhou. China J Antimicrob Chemother. 2016;71:2355-7.

9. World Health Organization (WHO). Global action plan to control the spread and impact of anti-microbial resistance in Neisseria gonorrhoeae. Geneva: WHO. Available from: http://apps.who.int/iris/bitstream/10665/44863/1/ 9789241503501_eng.pdf.

10. Kirkcaldy RD, Harvey A, Papp JR, Del Rio C, Soge OO, Holmes KK, Hook EW 3rd, Kubin G, Riedel S, Zenilman J, Pettus K, Sanders T, Sharpe S, Torrone E. Neisseria gonorrhoeae Antimicrobial Susceptibility Surveillance - The Gonococcal Isolate Surveillance Project, 27 Sites, United States, 2014 MMWR Surveill Summ. 2016:65:1-19.

11. Gu WM, Chen Y, Yang Y, Wu L, Hu WZ, YL J. Twenty-five-year changing pattern of gonococcal antimicrobial susceptibility in Shanghai: surveillance and its impact on treatment guidelines. BMC Infect Dis. 2014;14:731.

12. Cao W, Liang JY, Li XD, Bi C, Yang RD, Liang YH, et al. Trends in antimicrobial resistance in Neisseria gonorrhoeae isolated from Guangzhou, China, 2000 to 2005 and 2008 to 2013. Sex Transm Dis. 2015:42:27-9.

13. CLSI: Neisseria gonorrhoeae. In Performance Standards for Antimicrobial Susceptibility Testing; Twenty-Second Informational Supplement. CLSI document M100-S25. Wayne, PA: Clinical and Laboratory Standards Institute. 2015:80-82.

14. Breakpoint tables for interpretation of MICs and zone diameters: version 1.3. European Committee on Antimicrobial Susceptibility Testing. Available from: http://www.eucast.org/clinical_breakpoints/. Accessed January 15, 2012.

15. Sng EH, Yeo KL, Rajan VS. Simple method for detecting penicillinaseproducing Neisseria gonorrhoeae and Staphylococcus aureus. Br J Vener Dis. 1981:57:141-2.

16. Barry PM, Klausner JD. The use of cephalosporins for gonorrhea: the impending problem of resistance. Expert Opin Pharmacother. 2009;1 0:555-7.

17. Li S, Su XH, Le WJ, Jiang FX, Wang BX, Rice PA. Antimicrobial susceptibility of Neisseria gonorrhoeae isolates from symptomatic men attending the Nanjing sexually transmitted diseases clinic (2011-2012): genetic characteristics of isolates with reduced sensitivity to ceftriaxone. BMC Infect Dis. 2014;14:622.

18. Allen VG, Farrell DJ, Rebbapragada A, Tan J, Tijet N, Perusini SJ, et al. Molecular analysis of antimicrobial resistance mechanisms in Neisseria gonorrhoeae isolates from Ontario, Canada. Antimicrob Agents Chemother. 2011;55:703-12.

19. Liao M, Gu WM, Yang Y, Dillon JA. Analysis of mutations in multiple loci of Neisseria gonorrhoeae isolates reveals effects of PIB, PBP2 and MtrR on reduced susceptibility to ceftriaxone. J Antimicrob Chemother. 2011;66:1016-23.

20. Ng LK, Martin I, Liu G, Bryden L. Mutation in $23 \mathrm{~S}$ rRNA associated with macrolide resistance in Neisseria gonorrhoeae. Antimicrob Agents Chemother. 2002; 46:3020-5.

21. Martin IM, Ison CA, Aanensen DM, Fenton KA, Spratt BG. Rapid sequencebased identification of gonococcal transmission clusters in a large metropolitan area. J Infect Dis. 2004;189:1497-505.

22. Whiley DM, Limnios EA, Ray S, Sloots TP, Tapsall JW. Diversity of penA alterations and subtypes in Neisseria gonorrhoeae strains from Sydney, Australia, that are less susceptible to ceftriaxone. Antimicrob Agents Chemother. 2007:51:3111-6.

23. Martin I, Sawatzky P, Allen V, Hoang L, Lefebvre B, Mina N, et al. Emergence of Neisseria gonorrhoeae isolates with decreased susceptibilities to ceftriaxone and cefixime in Canada : 2001-2010. Sex Transm Dis. 2012;39:316-23.

24. Bi $\mathrm{P}$, Tong $\mathrm{S}$, Parton KA. Family self-medication and antibiotics abuse for children and juveniles in a Chinese city. Soc Sci Med. 2000;50:1445-50.
25. Shigemura K, Osawa K, Miura M, Tanaka K, Arakawa S, Shirakawa T, et al. Azithromycin resistance and its mechanism in Neisseria gonorrhoeae strains in Hyogo Japan. Antimicrob Agents Chemother. 2015;59:2695-9.

26. Cobo F, Cabezas-Fernández MT, Cabeza-Barrera MI. Antimicrobial susceptibility and typing of Neisseria gonorrhoeae strains from southern Spain, 2012-2014. Enferm Infecc Microbiol Clín. 2016;34:3-7.

27. Xue J, Ni C, Zhou H, Zhang C, van der Veen S. Occurrence of high-level azithromycin-resistant Neisseria gonorrhoeae isolates in China. J Antimicrob Chemother. 2015;70:3404-5.

28. Liang JY, Cao WL, Li XD, Bi C, Yang RD, Liang YH, et al. Azithromycinresistant Neisseria gonorrhoeae isolates in Guangzhou, China (2009-2013): coevolution with decreased susceptibilities to ceftriaxone and genetic characteristics. BMC Infect Dis. 2016;16:152.

29. Lynagh Y, Mac Aogáin M, Walsh A, Rogers TR, Unemo M, Crowley B. Detailed characterization of the first high-level azithromycin-resistant Neisseria gonorrhoeae cases in Ireland. J Antimicrob Chemother. 2015;70:2411-3.

30. Chisholm SA, Wilson J, Alexander S, Tripodo F, Al-Shahib A, Schaefer U, et al An outbreak of high-level azithromycin resistant Neisseria gonorrhoeae in England. Sex Transm Infect. 2015;92:365-7.

31. Bharat A, Demczuk W, Martin I, Mulvey MR. The effect of variants of penicillin-binding protein 2 (PBP2) on cephalosporin and carbapenem susceptibilities in Neisseria gonorrhoeae. Antimicrob Agents Chemother. 2015:59:5003-6.

32. Olsen B, Lan PT, Golparian D, Johansson E, Tran HK, Unemo M. Antimicrobial susceptibility and genetic characteristics of Neisseria gonorrhoeae isolates from Vietnam, 2011. BMC Infect Dis. 2013;13:40.

33. Whiley DM, Goire N, Lambert SB, Ray S, Limnios EA, Nissen MD, et al. Reduced susceptibility to ceftriaxone in Neisseria gonorrhoeae is associated with mutations G542S, P551S and P551L in the gonococcal penicillinbinding protein 2. J Antimicrobial Chemother. 2010;65:1615-8.

34. Unemo M, Golparian D, Nicholas R, Ohnishi M, Gallay A, Sednaoui P. Highlevel cefixime- and ceftriaxone-resistant N. gonorrhoeae in Europe (France): novel penA mosaic allele in a successful international clone causes treatment failure. Antimicrob. Agents Chemother. 2012:56:1273-80.

35. Chen SC, Yin YP, Dai XQ, Unemo M, Chen XS. First nationwide study regarding ceftriaxone resistance and molecular epidemiology of Neisseria gonorrhoeae in China. J Antimicrob Chemother. 2016;71:92-9.

\section{Submit your next manuscript to BioMed Central and we will help you at every step:}

- We accept pre-submission inquiries

- Our selector tool helps you to find the most relevant journal

- We provide round the clock customer support

- Convenient online submission

- Thorough peer review

- Inclusion in PubMed and all major indexing services

- Maximum visibility for your research

Submit your manuscript at www biomedcentral.com/submit
C) Biomed Central 